Теорія Ймовір. та Матем. Статист. Вип. 79, 2008
Theor. Probability and Math. Statist.

No. 79, 2009, Pages 73-81

S 0094-9000(09)00781-9

Article electronically published on December 29, 2009

\title{
ASYMPTOTIC PROPERTIES OF AN ESTIMATOR FOR THE DRIFT COEFFICIENT OF A STOCHASTIC DIFFERENTIAL EQUATION WITH FRACTIONAL BROWNIAN MOTION
}

UDC 519.21

\author{
E. I. KASYTS'KA AND P. S. KNOPOV
}

\begin{abstract}
A stochastic differential equation with respect to fractional Brownian motion is considered. We study the maximum likelihood estimator for the drift coefficient. We assume that the coefficient belongs to a given compact set of functions and prove the strong consistency of the estimator and its asymptotic normality.
\end{abstract}

Let $(\Omega, F, \mathrm{P})$ be a probability space and let a real stochastic process

$$
\{x(t), t \geq 0\}
$$

and a fractional Wiener process (fractional Brownian motion) $\{Z(t), t \geq 0\}$ be defined on $(\Omega, F, \mathrm{P})$, where $\mathrm{E} Z(t)=0, Z(0)=0$, and

$$
\mathrm{E}\{Z(t) Z(\tau)\}=\frac{1}{2}\left(t^{2 H}+\tau^{2 H}-|t-\tau|^{2 H}\right), \quad \frac{1}{2}<H<1 .
$$

Assume that a stochastic process $\{y(t), t \geq 0\}$ possesses the stochastic differential

$$
d y(t)=s_{0}(t) x(t) d t+d Z(t), \quad t \geq 0,
$$

where $s_{0}$ is a certain unknown function.

The problem considered in this paper is to estimate the function $s_{0}$ from the observations $\{(x(t), y(t)), 0 \leq t \leq T\}$.

Note that an analogous problem is considered in 1], where a standard Wiener process is substituted for $Z$ in equation (1).

\section{Consistency and the Asymptotic Distribution of estimators}

Below we list the main conditions to be imposed on the function $s_{0}$ and the stochastic processes $\{x(t), t \geq 0\}$ and $\{Z(t), t \geq 0\}$.

1. The function $s_{0}$ belongs to the family $K$ of all $2 \pi$-periodic functions $s: \mathbf{R} \rightarrow \mathbf{R}$ whose Fourier coefficients

$$
c_{k}(s)=\frac{1}{2 \pi} \int_{0}^{2 \pi} s(t) e^{i k t} d t, \quad k \in \mathbf{Z},
$$

are such that $\left|c_{0}(s)\right| \leq L$ and $\left|c_{k}(s)\right| \leq L|k|^{-a}, k \neq 0$, for some constants $L>0$ and $a>3$.

2000 Mathematics Subject Classification. Primary 60H10; Secondary 62M05.

Key words and phrases. Fractional Wiener process, stochastic integral, stochastic differential equation, drift coefficient. 
It is obvious that the functions of the family $K$ are continuously differentiable and that $K$ is a compact set with respect to the uniform convergence of functions.

For functions $s \in K$, we introduce the norm

$$
\|s\|=\left(\frac{1}{2 \pi} \int_{0}^{2 \pi} s^{2}(t) d t\right)^{1 / 2} .
$$

We say that $s_{0} \in K$ is an interior point of $K$ if

$$
\left|c_{0}\left(s_{0}\right)\right| \leq \tilde{L}, \quad\left|c_{k}\left(s_{0}\right)\right| \leq \frac{\tilde{L}}{|k|^{a}}, \quad k \neq 0,
$$

for some constant $\tilde{L}<L$.

2. The processes $\{x(t), t \geq 0\}$ and $\{Z(t), t \geq 0\}$ are independent.

3. There exists a constant $c>0$ such that

$$
\mathrm{E}\left\{(x(t))^{2}\right\} \leq c
$$

for all $t \geq 0$.

4. The trajectories of the process $\{x(t), t \geq 0\}$ are continuously differentiable with probability 1.

5. The process $\left\{(x(t))^{2}, t \geq 0\right\}$ is stationary in the wide sense.

Denote by $r(t)$ the covariance function of the process $\left\{(x(t))^{2}, t \geq 0\right\}$ :

$$
r(t)=\mathrm{E}\left\{\left((x(t))^{2}-\mathrm{E}(x(0))^{2}\right)\left((x(0))^{2}-\mathrm{E}(x(0))^{2}\right)\right\} .
$$

6. For some $L_{1}>0$ and $\gamma>0$,

$$
\int_{0}^{T}|r(t)| d t \leq L_{1} T^{1-\gamma}, \quad T>0 .
$$

Using conditions 2-4 and integration by parts [2], we define the following stochastic integral:

$$
\int_{a}^{b} s(\tau) x(\tau) d Z(\tau), \quad 0 \leq a \leq b,
$$

for an arbitrary continuously differentiable function $s: \mathbf{R} \rightarrow \mathbf{C}$ (where $\mathbf{C}$ is the set of complex numbers).

In what follows we need the following properties of the latter integral:

$$
\mathrm{E}\left\{\left[\int_{0}^{t} s(\tau) x(\tau) d Z(\tau)\right]^{2}\right\} \leq c_{1}\left(\int_{0}^{t}|s(\tau)|^{1 / H} d \tau\right)^{2 H}, \quad t>0
$$

where $c_{1}$ is a constant, and

$$
\begin{gathered}
\mathrm{E}\left\{\sup _{a \leq t \leq b}\left[\int_{a}^{t} s(\tau) x(\tau) d Z(\tau)\right]^{2}\right\} \\
0 \leq a<b
\end{gathered}
$$

where $c_{2}$ is another constant.

Note that the constants $c_{1}$ and $c_{2}$ depend on $H$.

To prove (2) and (3) one uses well-known properties of the stochastic integral with respect to a fractional Wiener process $(3,4])$ and the mutual independence of the processes $\{x(t), t \geq 0\}$ and $\{Z(t), t \geq 0\}$. 
With probability one, we have

$$
\begin{aligned}
\mathrm{E}\{ & {\left.\left[\int_{0}^{t} s(\tau) x(\tau) d Z(\tau)\right]^{2} / \sigma\{x(\tau), \tau \geq 0\}\right\} } \\
& =H(2 H-1) \int_{0}^{t} \int_{0}^{t} s\left(\tau_{1}\right) x\left(\tau_{1}\right) s\left(\tau_{2}\right) x\left(\tau_{2}\right)\left|\tau_{1}-\tau_{2}\right|^{2 H-2} d \tau_{1} d \tau_{2}
\end{aligned}
$$

Thus

$$
\begin{aligned}
\mathrm{E}\{[ & \left.\left.\int_{0}^{t} s(\tau) x(\tau) d Z(\tau)\right]^{2}\right\} \\
& =H(2 H-1) \int_{0}^{t} \int_{0}^{t} s\left(\tau_{1}\right) s\left(\tau_{2}\right) \mathrm{E}\left\{x\left(\tau_{1}\right) x\left(\tau_{2}\right)\right\}\left|\tau_{1}-\tau_{2}\right|^{2 H-2} d \tau_{1} d \tau_{2} \\
& \leq c \int_{0}^{t} \int_{0}^{t}\left|s\left(\tau_{1}\right)\right|\left|s\left(\tau_{2}\right)\right|\left|\tau_{1}-\tau_{2}\right|^{2 H-2} d \tau_{1} d \tau_{2} \\
& \leq c_{1}\left(\int_{0}^{t}|s(\tau)|^{1 / H} d \tau\right)^{2 H} .
\end{aligned}
$$

Note that the latter inequality is proved in 3 .

Therefore property (2) is proved.

Further, it is shown in [4] that, with probability 1 ,

$$
\begin{aligned}
\mathrm{E}\left\{\sup _{a \leq t \leq b}\left[\int_{a}^{t} s(\tau) x(\tau) d Z(\tau)\right]^{2} / \sigma\{x(\tau), \tau \geq 0\}\right\} \\
\leq c_{3}\left(\int_{a}^{b}|s(\tau) x(\tau)|^{1 / H} d \tau\right)^{2 H} \\
\quad \leq c_{3}\left(\left[\int_{a}^{b}\left(|s(\tau)|^{1 / H}\right)^{\alpha /(\alpha-1)} d \tau\right]^{(\alpha-1) / \alpha}\left[\int_{a}^{b}\left(|x(\tau)|^{1 / H}\right)^{\alpha} d \tau\right]^{1 / \alpha}\right)^{2 H} \\
\quad=c_{3}\left(\int_{a}^{b}|s(\tau)|^{2 /(2 H-1)} d \tau\right)^{2 H-1} \int_{a}^{b}|x(\tau)|^{2} d \tau
\end{aligned}
$$

via the Hölder inequality with $\alpha=2 H$ and $\beta=2 H /(2 H-1)$.

Then

$$
\begin{aligned}
\mathrm{E}\left\{\sup _{a \leq t \leq b}\left[\int_{a}^{t} s(\tau) x(\tau) d Z(\tau)\right]^{2}\right\} & \leq c_{3}\left(\int_{a}^{b}|s(\tau)|^{2 /(2 H-1)} d \tau\right)^{2 H-1} \int_{a}^{b} \mathrm{E}\left\{|x(\tau)|^{2}\right\} d \tau \\
& \leq c_{2}(b-a)\left(\int_{a}^{b}|s(\tau)|^{\frac{2}{2 H-1}} d \tau\right)^{2 H-1}
\end{aligned}
$$

Hence property (3) is proved too.

We turn to the estimation of the function $s_{0}$. Consider the estimator defined as the point of maximum of the functional

$$
Q_{T}(s)=\frac{1}{T} \int_{0}^{T} s(t) x(t) d y(t)-\frac{1}{2 T} \int_{0}^{T} s^{2}(t) x^{2}(t) d t, \quad s \in K .
$$

This estimator exists with probability one. Denote by $s_{T}$ an arbitrary point of maximum of (41). As in the paper [5], the properties of the family $K$ imply that the function $s_{T}(t, \omega)$ can be chosen to be a separable measurable process. 
Lemma 1.1. Let conditions 1-4 hold. Then

$$
\mathrm{P}\left\{\lim _{T \rightarrow \infty} \max _{s \in K}\left|\frac{1}{T} \int_{0}^{T} s(t) x(t) d Z(t)\right|=0\right\}=1 .
$$

Proof. Put

$$
\eta_{T}=\max _{s \in K}\left|\frac{1}{T} \int_{0}^{T} s(t) x(t) d Z(t)\right| .
$$

Expanding $s$ in the Fourier series we obtain

$$
\begin{aligned}
\mathrm{E}\left\{\left(\eta_{T}\right)^{2}\right\} & =\mathrm{E}\left\{\max _{s \in K}\left(\frac{1}{T} \sum_{k=-\infty}^{\infty}\left[c_{k}(s) \int_{0}^{T} e^{i k t} x(t) d Z(t)\right]\right)^{2}\right\} \\
& \leq \mathrm{E}\left\{\left(\frac{1}{T} \sum_{k=-\infty}^{\infty}\left[\frac{L}{|k|^{a}}\left|\int_{0}^{T} e^{i k t} x(t) d Z(t)\right|\right]\right)^{2}\right\} .
\end{aligned}
$$

By definition, the denominator of the term corresponding to $k=0$ in the latter sum (and in similar sums throughout below) is equal to 1 but not $|k|^{a}$.

Applying the Cauchy-Bunyakovskil inequality and the first property of the stochastic integral, we derive from relation (5) that

$$
\mathrm{E}\left\{\left(\eta_{T}\right)^{2}\right\} \leq\left(\frac{1}{T} \sum_{k=-\infty}^{\infty} \frac{L}{|k|^{a}}\left[\mathrm{E}\left|\int_{0}^{T} e^{i k t} x(t) d Z(t)\right|^{2}\right]^{1 / 2}\right)^{2} \leq \frac{c_{4}}{T^{2(1-H)}}
$$

where $c_{4}=c_{1} L^{2}\left(\sum_{k=-\infty}^{\infty}|k|^{-a}\right)^{2}$.

It is clear that there exists a positive integer number $p$ such that $2 p(1-H)>1$. Consider the sequence $T(n)=n^{p}, n \in \mathbf{N}$. According to bound (6) and the BorelCantelli lemma,

$$
\mathrm{P}\left(\lim _{n \rightarrow \infty} \eta_{T(n)}=0\right)=1
$$

For $T_{0} \geq 1$,

$$
\sup _{T>T_{0}} \eta_{T} \leq \sup _{n: T(n+1)>T_{0}} \sup _{T(n) \leq T \leq T(n+1)} \eta_{T}
$$

For all $n$,

$$
\begin{aligned}
\sup _{T(n) \leq T \leq T(n+1)} & \eta_{T}=\eta_{T(n)}+\sup _{T(n) \leq T \leq T(n+1)}\left(\eta_{T}-\eta_{T(n)}\right) \\
\leq & \eta_{T(n)} \\
& \quad+\sup _{T(n) \leq T \leq T(n+1)} \max _{s \in K}\left|\frac{1}{T} \int_{0}^{T} s(t) x(t) d Z(t)-\frac{1}{T(n)} \int_{0}^{T(n)} s(t) x(t) d Z(t)\right| \\
\leq & \eta_{T(n)}+\frac{T(n+1)-T(n)}{(T(n))^{2}} \max _{s \in K}\left|\int_{0}^{T(n)} s(t) x(t) d Z(t)\right| \\
& +\frac{1}{T(n)} \sup _{T(n) \leq T \leq T(n+1)} \max _{s \in K}\left|\int_{T(n)}^{T} s(t) x(t) d Z(t)\right| \\
= & \frac{T(n+1)}{T(n)} \eta_{T(n)}+\zeta_{n},
\end{aligned}
$$


where

We have

$$
\zeta_{n}=\frac{1}{T(n)} \sup _{T(n) \leq T \leq T(n+1)} \max _{s \in K}\left|\int_{T(n)}^{T} s(t) x(t) d Z(t)\right| .
$$

$$
\frac{T(n+1)}{T(n)}=\left(1+\frac{1}{n}\right)^{p} \rightarrow 1, \quad n \rightarrow \infty .
$$

Taking into account equality (7) we get

$$
\mathrm{P}\left\{\lim _{n \rightarrow \infty} \frac{T(n+1)}{T(n)} \eta_{T(n)}=0\right\}=1 .
$$

Performing elementary transformations and using properties of the stochastic integral, we obtain

$$
\begin{aligned}
& \mathrm{E}\left\{\left(\zeta_{n}\right)^{2}\right\} \leq \frac{1}{(T(n))^{2}} \mathrm{E}\left(\sum_{k=-\infty}^{\infty}\left[\frac{L}{|k|^{a}} \sup _{T(n) \leq T \leq T(n+1)}\left|\int_{T(n)}^{T} e^{i k t} x(t) d Z(t)\right|\right]\right)^{2} \\
& \leq \frac{1}{(T(n))^{2}}\left(\sum_{k=-\infty}^{\infty}\left[\frac{L}{|k|^{a}}\left(\mathrm{E}\left\{\sup _{T(n) \leq T \leq T(n+1)}\left|\int_{T(n)}^{T} e^{i k t} x(t) d Z(t)\right|^{2}\right\}\right)^{1 / 2}\right]\right)^{2} \\
& \leq \frac{(T(n+1)-T(n))^{2 H}}{(T(n))^{2}} c_{2} L^{2}\left(\sum_{k=-\infty}^{\infty} \frac{1}{|k|^{a}}\right)^{2} \\
& \quad=\frac{c_{5}}{n^{2 p(1-H)}}\left(\left(1+\frac{1}{n}\right)^{p}-1\right)^{2 H} \\
& \leq \frac{c_{6}}{n^{2 p(1-H)}}
\end{aligned}
$$

where $c_{5}$ and $c_{6}$ are some constants. This implies that

$$
\mathrm{P}\left(\lim _{n \rightarrow \infty} \zeta_{n}=0\right)=1 .
$$

Now the lemma follows from relations (8)-(11).

Remark 1.1. If the assumptions of Lemma 1.1 hold, then

$$
\mathrm{P}\left\{\lim _{T \rightarrow \infty} \max _{s \in K}\left|\frac{1}{T} \int_{0}^{T}\left(s(t)-s_{0}(t)\right) x(t) d Z(t)\right|=0\right\}=1 .
$$

Lemma $1.2([1])$. Let $\{\xi(t), t \in \mathbf{R}\}$ be a real wide sense stationary stochastic process with mean $\mathrm{E} \xi(t)=0$ and whose covariance function $r(t)=\mathrm{E}\{\xi(t) \xi(0)\}, t \in \mathbf{R}$, is such that

$$
\int_{0}^{T}|r(t)| d t \leq L T^{1-\gamma}
$$

for all $T>0$ and some positive numbers $L$ and $\gamma$. Then

$$
\mathrm{P}\left\{\lim _{T \rightarrow \infty} \sup _{s \in K}\left|\frac{1}{T} \int_{0}^{T} s(t) \xi(t) d t\right|=0\right\}=1 .
$$

Remark 1.2 (1]). Lemma 1.2 remains true if the difference of two functions of the family $K$ is substituted for $s \in K$ in the above integral.

Remark 1.3. Lemma 1.2 holds also for the square of the difference of two functions of the family $K$. 
Remark 1.3 can be proved by an observation that the square of the difference of two functions belonging to $K$ can be used in the proof of Lemma 2.2 in [1].

Theorem 1.1. Let the assumptions of Lemma 1.1 as well as conditions 5 and 6 hold. Then

$$
\mathbf{P}\left\{\lim _{T \rightarrow \infty} \sup _{t \in \mathbf{R}}\left|s_{T}(t)-s_{0}(t)\right|=0\right\}=1 .
$$

Proof. Note that

$$
\begin{aligned}
Q_{T}\left(s_{T}\right)-Q_{T}\left(s_{0}\right)= & \frac{1}{T} \int_{0}^{T}\left(s_{T}(t)-s_{0}(t)\right) x(t) d Z(t) \\
& -\frac{1}{2 T} \int_{0}^{T}\left(s_{T}(t)-s_{0}(t)\right)^{2}(x(t))^{2} d t .
\end{aligned}
$$

By the definition of the estimator $s_{T}$,

$$
Q_{T}\left(s_{T}\right) \geq Q_{T}\left(s_{0}\right) .
$$

Then

$$
\begin{aligned}
\max _{s \in K} \mid & \frac{1}{T} \int_{0}^{T}\left(s(t)-s_{0}(t)\right) x(t) d Z(t) \mid \\
& +\max _{s \in K}\left|\frac{1}{2 T} \int_{0}^{T}\left(s_{T}(t)-s_{0}(t)\right)^{2}\left[(x(t))^{2}-\mathrm{E}\left\{(x(0))^{2}\right\}\right] d t\right| \\
\geq & \frac{1}{2 T} \mathrm{E}\left\{(x(0))^{2}\right\} \int_{0}^{T}\left(s_{T}(t)-s_{0}(t)\right)^{2} d t
\end{aligned}
$$

by equality (12).

Lemmas 1.1 and 1.2 together with Remarks 1.1 and 1.3 and relation (13) imply that

$$
\mathrm{P}\left\{\lim _{T \rightarrow \infty} \frac{1}{T} \int_{0}^{T}\left(s_{T}(t)-s_{0}(t)\right)^{2} d t=0\right\}=1,
$$

whence

$$
\mathrm{P}\left(\lim _{T \rightarrow \infty}\left\|s_{T}-s_{0}\right\|=0\right)=1 .
$$

Now relation (15) yields Theorem 1.1 .

In what follows we will make use of the following conditions.

7. The process $\{x(t), t \geq 0\}$ is equal to 1 for all $t$.

8. Let a function $\varphi \in K$ be such that

1) $\frac{1}{2 \pi} \int_{0}^{2 \pi} \varphi^{2}(t) d t=1$,

2) $\lim _{T \rightarrow \infty} H(2 H-1) T^{-2 H} \int_{0}^{T} \int_{0}^{T} \varphi\left(t_{1}\right) \varphi\left(t_{2}\right)\left|t_{1}-t_{2}\right|^{2 H-2} d t_{1} d t_{2}=\Delta$.

9. The function $s_{0}$ is an interior point of the set $K$.

Theorem 1.2. Let conditions 1 and 7-9 hold. Then the random variable

$$
\frac{T^{1-H}}{2 \pi} \int_{0}^{2 \pi} \varphi(t)\left(s_{T}(t)-s_{0}(t)\right) d t
$$

converges in distribution as $T \rightarrow \infty$ to the Gaussian law $N(0, \Delta)$ with mean 0 and variance $\Delta$. 
Proof. By Theorem 1.1, the function $s_{T}$ is an interior point of the family $K$ with probability converging to 1 as $T \rightarrow \infty$. It is easy to show that the functional $Q_{T}$ is differentiable at the point $s_{T}$ with the same probability. Indeed, we evaluate the weak differential of $Q_{T}$ in some neighborhood of $s_{T}$ as follows:

$$
\begin{aligned}
D Q_{T}(s, h) & =\left.\frac{d}{d \varepsilon} Q_{T}(s+\varepsilon h)\right|_{\varepsilon=0} \\
& =\frac{1}{T} \int_{0}^{T} h(t) d Z(t)+\frac{1}{T} \int_{0}^{T} h(t) s_{0}(t) d t-\frac{1}{T} \int_{0}^{T} h(t) s(t) d t .
\end{aligned}
$$

Properties of the differential $D Q_{T}(s, h)$ imply that the strong differential of $Q_{T}$ at the point $s_{T}$ exists and it coincides with the weak differential [6].

By the necessary condition for the existence of an extremum,

$$
D Q_{T}\left(s_{T}, \varphi\right)=0
$$

with probability converging to 1 as $T \rightarrow \infty$.

Thus, with the same probability,

$$
\frac{1}{T} \int_{0}^{T} \varphi(t) d Z(t)+\frac{1}{T} \int_{0}^{T} \varphi(t)\left(s_{0}(t)-s_{T}(t)\right) d t=0
$$

as $T \rightarrow \infty$.

We add to and subtract from the left hand side of (16) the following expression:

$$
\frac{1}{2 \pi} \int_{0}^{2 \pi} \varphi(t)\left(s_{T}(t)-s_{0}(t)\right) d t \frac{1}{T} \int_{0}^{T} \varphi^{2}(t) d t
$$

Note that

$$
\begin{aligned}
-\frac{1}{T} & \int_{0}^{2 \pi\left[\frac{T}{2 \pi}\right]} \varphi(t)\left(s_{T}(t)-s_{0}(t)\right) d t+\frac{1}{2 \pi} \int_{0}^{2 \pi} \varphi(t)\left(s_{T}(t)-s_{0}(t)\right) d t \frac{1}{T} \int_{0}^{2 \pi\left[\frac{T}{2 \pi}\right]} \varphi^{2}(t) d t \\
= & -\frac{1}{T}\left[\frac{T}{2 \pi}\right] \int_{0}^{2 \pi} \varphi(t)\left(s_{T}(t)-s_{0}(t)\right) d t \\
& +\frac{1}{2 \pi} \int_{0}^{2 \pi} \varphi(t)\left(s_{T}(t)-s_{0}(t)\right) d t \frac{1}{T}\left[\frac{T}{2 \pi}\right] \int_{0}^{2 \pi} \varphi^{2}(t) d t \\
= & 0 .
\end{aligned}
$$

Thus

$$
\begin{aligned}
\frac{1}{T} \int_{0}^{T} & \varphi(t) d Z(t)+\frac{1}{T} \int_{2 \pi\left[\frac{T}{2 \pi}\right]}^{T} \varphi(t)\left(s_{0}(t)-s_{T}(t)\right) d t \\
& +\frac{1}{2 \pi} \int_{0}^{2 \pi} \varphi(t)\left(s_{T}(t)-s_{0}(t)\right) d t \frac{1}{T} \int_{2 \pi\left[\frac{T}{2 \pi}\right]}^{T} \varphi^{2}(t) d t \\
& \quad-\frac{1}{2 \pi} \int_{0}^{2 \pi} \varphi(t)\left(s_{T}(t)-s_{0}(t)\right) d t \frac{1}{T} \int_{0}^{T} \varphi^{2}(t) d t \\
= & 0
\end{aligned}
$$


with probability converging to 1 as $T \rightarrow \infty$. Hence, with the same probability,

$$
\begin{aligned}
T^{1-H} & \frac{1}{2 \pi} \int_{0}^{2 \pi} \varphi(t)\left(s_{T}(t)-s_{0}(t)\right) d t \\
= & \left(\frac{1}{T} \int_{0}^{T} \varphi^{2}(t) d t\right)^{-1} \\
& \times\left(\frac{1}{T^{H}} \int_{0}^{T} \varphi(t) d Z(t)+\frac{1}{T^{H}} \int_{2 \pi\left[\frac{T}{2 \pi}\right]}^{T} \varphi(t)\left(s_{0}(t)-s_{T}(t)\right) d t\right. \\
& \left.\quad+\frac{1}{2 \pi} \int_{0}^{2 \pi} \varphi(t)\left(s_{T}(t)-s_{0}(t)\right) d t \frac{1}{T^{H}} \int_{2 \pi\left[\frac{T}{2 \pi}\right]}^{T} \varphi^{2}(t) d t\right) .
\end{aligned}
$$

Consider

$$
\frac{1}{T} \int_{0}^{T} \varphi^{2}(t) d t=\frac{1}{T}\left(\int_{0}^{2 \pi\left[\frac{T}{2 \pi}\right]} \varphi^{2}(t) d t+\int_{2 \pi\left[\frac{T}{2 \pi}\right]}^{T} \varphi^{2}(t) d t\right)
$$

Then

$$
\begin{aligned}
\frac{1}{T} \int_{0}^{2 \pi\left[\frac{T}{2 \pi}\right]} \varphi^{2}(t) d t & =\frac{1}{T}\left[\frac{T}{2 \pi}\right] \int_{0}^{2 \pi} \varphi^{2}(t) d t=\frac{1}{T}\left[\frac{T}{2 \pi}\right] 2 \pi \rightarrow 1, \quad T \rightarrow \infty, \\
& \frac{1}{T} \int_{2 \pi\left[\frac{T}{2 \pi}\right]}^{T} \varphi^{2}(t) d t \rightarrow 0, \quad T \rightarrow \infty .
\end{aligned}
$$

Hence

$$
\frac{1}{T} \int_{0}^{T} \varphi^{2}(t) d t \rightarrow 1, \quad T \rightarrow \infty .
$$

Since the functions of the family $K$ are bounded, the second and third terms in the expression on the right hand side of (17) converge to 0 with probability 1 as $T \rightarrow \infty$.

Now we study the random variable

$$
\xi_{T}=\frac{1}{T^{H}} \int_{0}^{T} \varphi(t) d Z(t) .
$$

Its distribution is normal [3] with mean 0 and variance

$$
\frac{H(2 H-1)}{T^{2 H}} \int_{0}^{T} \int_{0}^{T} \varphi\left(t_{1}\right) \varphi\left(t_{2}\right)\left|t_{1}-t_{2}\right|^{2 H-2} d t_{1} d t_{2} .
$$

The assumptions of the theorem and properties of Gaussian random variables imply that $\xi_{T}$ weakly converges to $N(0, \Delta)$ as $T \rightarrow \infty$.

Taking into account (18) and the preceding reasoning, we prove that the right hand side of equality (17) converges in distribution to $N(0, \Delta)$ as $T \rightarrow \infty$. This completes the proof of Theorem 1.2 .

\section{Concluding Remarks}

The results concerning the asymptotic behavior of the estimator of the drift diffusion of a stochastic differential equation with respect to fractional Brownian motion obtained above imply that the proposed estimators are optimal, and this allows one to use them for solving various applied problems. 


\section{BIBLIOGRAPHY}

1. A. Ya. Dorogovtsev, The Theory of Estimates of the Parameters of Random Processes, Vyshcha Shkola, Kiev, 1982. (Russian) MR668517 (84h:62122)

2. M. Zähle, Integration with respect to fractal functions and stochastic calculus, Probab. Theory Relat. Fields III (1998), 333-374. MR 1640795 (99j:60073)

3. J. Mémin, Yu. Mishura, and E. Valkeila, Inequalities for the moments of Wiener integrals with respect to a fractional Brownian motion, Statist. Probab. Lett. 51 (2001), 197-206. MR1822771 (2002b:60096)

4. Yu. V. Krvavych and Yu. S. Mishura, Some maximal inequalities for moments of Wiener integrals with respect to fractional Brownian motion, Teor. Imovir. Mat. Stat. 61 (1999), 72-83; English transl. in Theory Probab. Math. Statist. 61 (2000), 75-86. MR.1866968(2002h:60072)

5. J. Pfanzagl, On the measurability and consistency of minimum contrast estimates, Metrika $\mathbf{1 4}$ (1969), 249-272.

6. A. N. Kolmogorov and S. V. Fomin, Elements of the Theory of Functions and Functional Analysis, Nauka, Moscow, 1968; English transl. from the first (1960) Russian ed., Graylock Press, Albany, N.Y., 1961. MR0234241 (38:2559)

Glushkov Institute for Cybernetics, National Academy of Sciences of Ukraine, AcademiCian Glushkov Avenue, 03187 Kyiv, Ukraine

Glushkov Institute for Cybernetics, National Academy of Sciences of Ukraine, AcademiCian Glushkov Avenue, 03187 Kyiv, Ukraine

E-mail address: knopov1@yahoo.com

Received $7 / \mathrm{JUL} / 2008$

Translated by OLEG KLESOV 